University of Nebraska - Lincoln

DigitalCommons@University of Nebraska - Lincoln

Faculty Publications, Department of Physics and Astronomy

Research Papers in Physics and Astronomy

$12-2005$

Focused-Laser Interferometric Position Sensor

Stephen J. Friedman

University of Nebraska - Lincoln

Brett E. Barwick

University of Nebraska-Lincoln, brett.barwick@trincoll.edu

Herman Batelaan

University of Nebraska - Lincoln, hbatelaan@unl.edu

Follow this and additional works at: https://digitalcommons.unl.edu/physicsfacpub

Part of the Physics Commons

Friedman, Stephen J.; Barwick, Brett E.; and Batelaan, Herman, "Focused-Laser Interferometric Position Sensor" (2005). Faculty Publications, Department of Physics and Astronomy. 116.

https://digitalcommons.unl.edu/physicsfacpub/116

This Article is brought to you for free and open access by the Research Papers in Physics and Astronomy at DigitalCommons@University of Nebraska - Lincoln. It has been accepted for inclusion in Faculty Publications, Department of Physics and Astronomy by an authorized administrator of DigitalCommons@University of Nebraska Lincoln. 


\title{
Focused-laser interferometric position sensor
}

\author{
Stephen J. Friedman, Brett Barwick, and Herman Batelaan \\ Department of Physics and Astronomy, University of Nebraska-Lincoln, Lincoln, Nebraska 68588-0111
}

(Received 13 July 2005; accepted 2 October 2005; published online 21 December 2005)

\begin{abstract}
We describe a simple method to measure the position shifts of an object with a range of tens of micrometers using a focused-laser (FL) interferometric position sensor. In this article we examine the effects of mechanical vibration on FL and Michelson interferometers. We tested both interferometers using vibration amplitudes ranging from 0 to $20 \mu \mathrm{m}$. Our FL interferometer has a resolution much better than the diffraction grating periodicities of 10 and $14 \mu \mathrm{m}$ used in our experiments. A FL interferometer provides improved mechanical stability at the expense of spatial resolution. Our experimental results show that Michelson interferometers cannot be used when the vibration amplitude is more than an optical wavelength. The main purpose of this article is to demonstrate that a focused-laser interferometric position sensor can be used to measure the position shifts of an object on a less sensitive, micrometer scale when the vibration amplitude is too large to use a Michelson interferometer. (C) 2005 American Institute of Physics. [DOI: 10.1063/1.2130667]
\end{abstract}

\section{INTRODUCTION}

Optical interferometers have been used for over 140 years in scientific measurements. Early interferometers were devised and suggested by Fizeau in the 1860 s. ${ }^{1-3}$ In the early 1880s Michelson created the interferometer that bears his name. ${ }^{4,5}$ Other configurations such as the Mach-Zehnder, Fabry-Perot, and Sagnac interferometers were subsequently developed. ${ }^{6-10}$ Interferometers can be used to make extremely accurate measurements of length. For example, one can detect the longitudinal (axial) position shifts of a movable mirror in a Michelson interferometer with an accuracy much better than the optical wavelength $\lambda_{\text {opt. }}{ }^{11}$ If this mirror is attached to an object, then monitoring the interference fringes will indicate the longitudinal position of that object with an accuracy also much better than $\lambda_{\text {opt }}$. Other interferometric methods have been proposed to measure longitudinal position shifts with high spatial resolution. ${ }^{12,13}$ However, when measuring the position of a vibrating object (for example, inside a mechanically pumped vacuum chamber), these interferometers are often too sensitive. If the vibration amplitude is too large it smears out the interference fringes. Acoustic position sensors have been proposed and used as one method to measure an object's position on a less sensitive scale. ${ }^{14}$ However, this method does not work in a vacuum chamber.

Another method to measure position shifts on a less sensitive scale is to monitor the interference fringes of a threegrating Mach-Zehnder (MZ) interferometer. ${ }^{15}$ The spatial resolution of a three-grating MZ interferometer is determined by the periodicity of the diffraction gratings used in the interferometer. By attaching the third (position sensing) grating to an object, one can use the MZ interference fringes to measure the transverse position shifts of that object. A drawback of using a three-grating MZ configuration for this purpose is that alignment is difficult. Charge-coupled device (CCD)-based optical position sensors (COPS) provide another way to measure transverse position shifts, but the $2 \mu \mathrm{m}$ resolution of these sensors is typically worse than the resolution of a three-grating MZ interferometer. ${ }^{16}$

We demonstrate a simple interferometric configuration that shares with the three-grating MZ interferometer the ability to measure transverse position shifts on a less sensitive scale but is much easier to align and use. This configuration involves focusing a laser beam close to a diffraction grating which causes the diffraction orders to partially overlap in the far field. The overlap produces interference fringes which can be scanned by moving the grating in a transverse direction relative to the incident laser beam. As the grating moves, the fringes oscillate, and the oscillation period is determined by the grating constant (periodicity) $d$. This is unlike a Michelson interferometer where, as a mirror is moved, the oscillation period of the fringes is $\lambda_{\text {opt }} / 2$. If the grating in a focused-laser (FL) interferometer is attached to an object, the interference fringes can be used as an indicator of the relative position of that object. The primary advantages of using a FL interferometric position sensor are the following: (1) it is much less sensitive to mechanical vibration than a Michelson interferometer; (2) it has the same spatial resolution as a three-grating MZ interferometer but is much easier to align; and (3) the working distance of a FL position sensor can be easily adjusted. We successfully used the FL technique inside a (vibrating) mechanically pumped vacuum chamber. The data presented in this article were taken outside of vacuum using transmission gratings with periodicities of $d=10 \mu \mathrm{m}$ and $d=14 \mu \mathrm{m}$. Our FL interferometer was operated concurrently with a Michelson interferometer to allow a detailed comparison of the effects of mechanical vibration on both interferometers.

\section{EXPERIMENTAL APPARATUS}

The experimental apparatus to compare our FL interferometer to a Michelson interferometer consists of a $30 \mathrm{~mW}$ diode pumped Nd:YAG (yttrium aluminum garnet) laser $\left(\lambda_{\text {opt }}=532 \mathrm{~nm}\right)$, a spherical lens $(f=1 \mathrm{~cm})$, a cylindrical lens 
(a) Focused-laser and Michelson interferometers

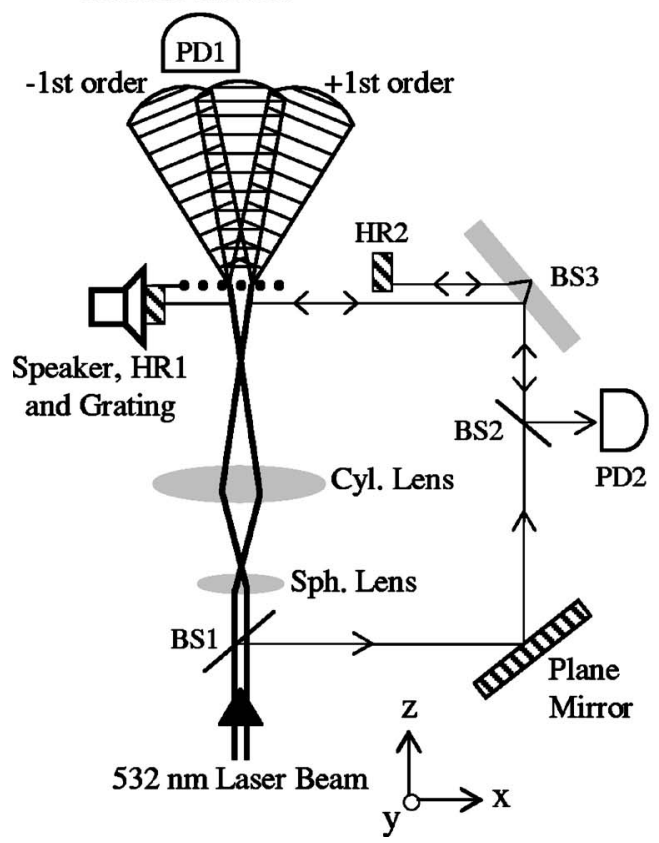

(b) Focused-laser interferometer using a reflective grating

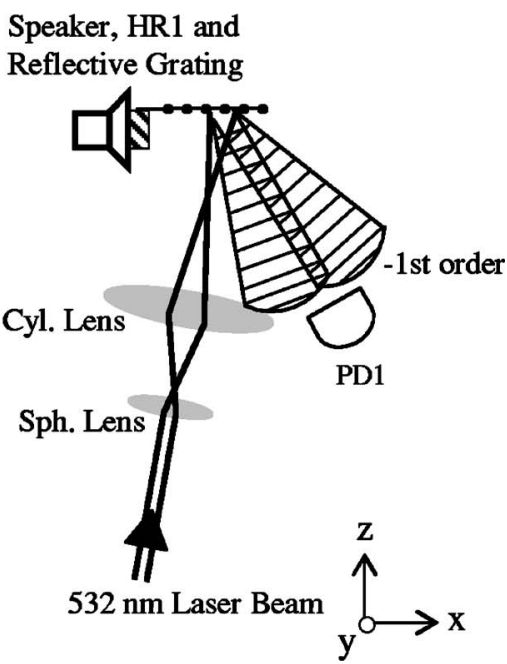

FIG. 1. Experimental schematics (not to scale): (a) concurrent focused-laser (FL) and Michelson interferometers and (b) FL interferometer configuration using a reflective diffraction grating showing only the zeroth and first diffraction orders.

$(f=10 \mathrm{~cm})$, three beam splitters (BS), a plane mirror, two $98.5 \%$ reflective mirrors (HR) coated at $500 \mathrm{~nm}$, a transmission diffraction grating (Edmund Optics crown glass grating G46-067, Industrial Fiber Optics photographic film grating), a small audio speaker, and two Thorlabs DET210 photodetectors (PD). Alternatively, a $\mathrm{He}-\mathrm{Ne}$ laser can be used. The entire experimental apparatus is mounted on an optical table - a schematic of the components is shown in Fig. 1(a). The schematic in Fig. 1(b) shows how a reflective diffraction grating one dimensional (1D) or two dimensional (2D) can be used in a FL interferometer configuration.

The mirror HR1 is glued to the dust cap of the audio speaker. HR1 is semicircular shaped with a radius and thickness of $0.25 \mathrm{in}$. The small size was chosen to minimize the load on the speaker membrane. The transmission grating is glued to the edge of HR1. By modulating the speaker, we can simultaneously modulate the position of HR1 and the grating. The modulating signal can be combined with a second signal to introduce mechanical vibration into the speaker modulation. The spherical lens is used to expand the incident laser beam (1 mm in diameter) to a diameter of about $1 \mathrm{~cm}$ at the position of the cylindrical lens. The cylindrical lens focuses the laser beam approximately $3 \mathrm{~mm}$ away from the diffraction grating. The cylindrical lens is located about $15 \mathrm{~cm}$ away from the grating, and we call this the working distance of the FL position sensor. The working distance can be easily adjusted by changing the focal lengths and/or the configuration of the lenses. To create our FL interferometer, we position the lenses so that the laser beam divergence is larger than the diffraction angle of roughly $40 \mathrm{mrad}$. This causes the diffraction orders to partially overlap in the far field, and we observe interference fringes on a screen (see Fig. 2). The photodetector PD1 is positioned in the overlapping region to measure the signal of the FL interferometer fringes.

To monitor the position of the grating in a conventional way, the mirror HR1 is used in one of the arms of a Michelson interferometer. We split off a small fraction of the incident laser beam at the beam splitter BS1 to make a Michelson interferometer shown in Fig. 1(a). Neutral density filters are used to balance the laser intensities in the two arms of the Michelson, and the Michelson fringes are measured with the signal from photodetector PD2. We observe the interference fringes of both interferometers on a digital oscilloscope (Tektronix TDS 3034) as the position of HR1 and

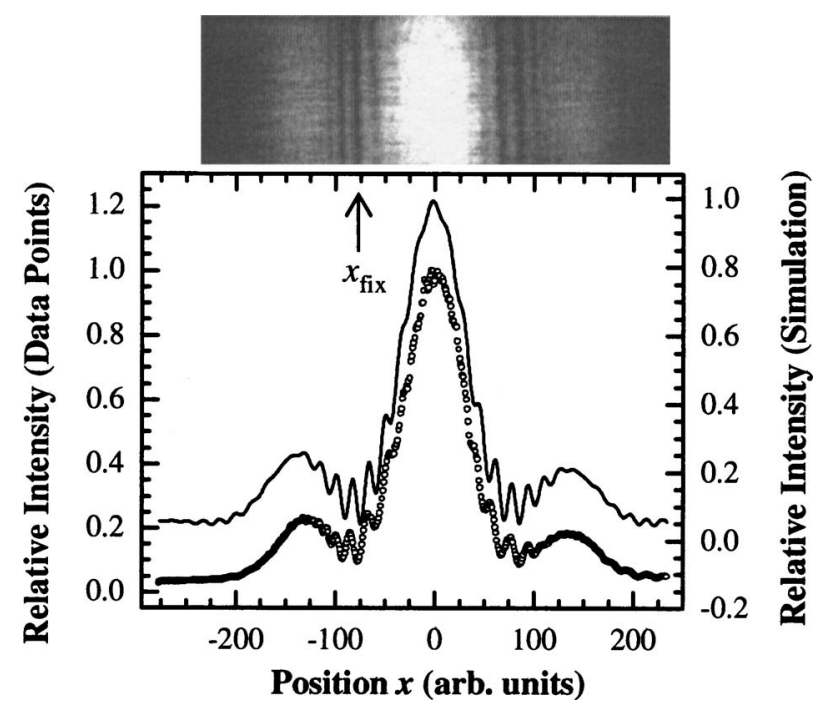

FIG. 2. CCD image of a typical diffraction pattern and interference fringes created by a FL interferometer, and the relative intensities of this CCD image plotted on the same horizontal scale. The round symbols are integrated relative intensities of the CCD image as a function of position $x$, using the vertical scale labeled on the left axis. The solid line is a numerical simulation of the data using optical diffraction theory-it is offset vertically by 0.2 to make the graph easier to view and corresponds to the vertical scale labeled on the right axis. The arrow indicates the fixed position $\left(x_{\mathrm{fix}}\right)$ of photodetector PD1 to measure the interference signals of the FL interferometer. 
(a) Modulation $=3 \mathrm{~Hz}$ sawtooth

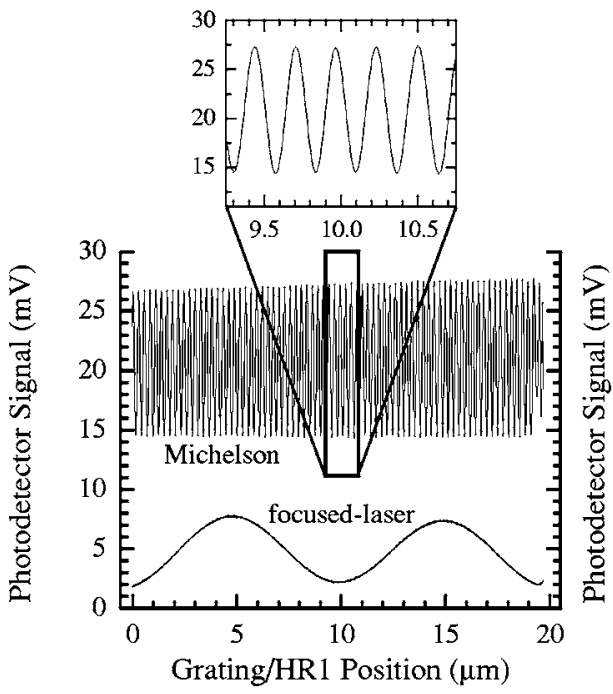

(b) Modulation = $3 \mathrm{~Hz}$ sawtooth + white noise

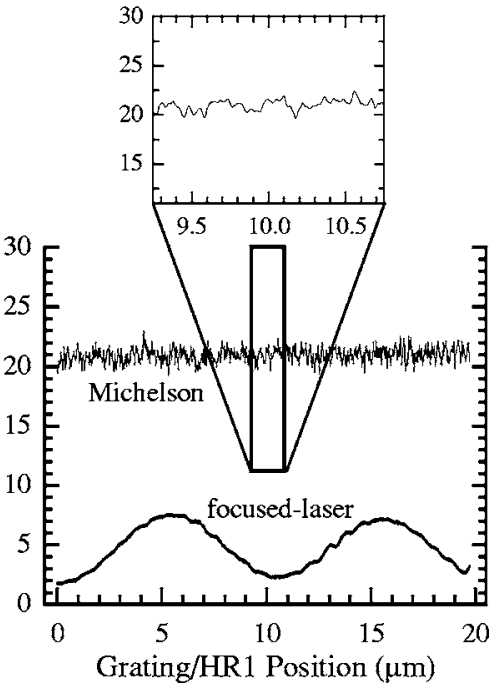

FIG. 3. Spectra of Michelson interferometer fringes and focused-laser interferometer fringes using a photographic film diffraction grating with a periodicity of $d=10 \mu \mathrm{m}$. The insets show an expanded view of the indicated region of Michelson fringes. (a) Plots with a $3 \mathrm{~Hz}$ triangular wave (sawtooth) modulation of the grating and mirror HR1 and (b) plots with $0.75 \mathrm{~V}$ (rms amplitude) of white noise added to the $3 \mathrm{~Hz}$ modulation. the diffraction grating are modulated with the speaker. Since we use diffraction gratings with periodicities of about $10 \mu \mathrm{m}$ in our experiments, our FL interferometer is much less sensitive to mechanical vibration than our Michelson interferometer. Tapping the optical table does not affect the FL fringes noticeably, but it significantly deforms the Michelson fringes. We examined the sensitivity of both interferometers to mechanical vibration by adding a second signal to the speaker modulation.

\section{CCD IMAGE OF INTERFERENCE FRINGES}

The image in Fig. 2 illustrates a typical diffraction pattern and interference fringes created by our FL interferometer. We used a crown glass transmission grating with 70 grooves $/ \mathrm{mm}(d=14 \mu \mathrm{m})$ to create this diffraction pattern. We captured the image in the figure using a chargecoupled device camera (SBIG ST-9E) and a 28-100 mm zoom lens (Nikon). Well-resolved interference fringes are present in the regions where the zeroth and first orders overlap. The round symbols in Fig. 2 are integrated (relative) intensities of the CCD image as a function of position $x$ at the detection screen. In the figure, the data and the CCD image are shown on the same horizontal scale.

The solid line in Fig. 2 is a numerical simulation of the data using optical diffraction theory. ${ }^{17}$ We modeled the illumination of a blazed transmission grating with a nonplanar (divergent) incident wave function. We then took the Fourier transform of the wave function $E\left(x^{\prime}\right)$ emerging from the grating to obtain $E\left(k_{x^{\prime}}\right)$. Here $x^{\prime}$ is the position at the grating and $k_{x^{\prime}}$ is its corresponding conjugate variable. The relative intensities of the simulation in Fig. 2 correspond to the distribution $|E(x)|^{2}$. The position $x$ at the screen is given by $x$ $=L \tan \theta$, for $\theta=\sin ^{-1}\left(k_{x} / k\right)$. Here $L$ is the distance between the grating and the detection screen and $k=2 \pi / \lambda_{\text {opt }}$ is the magnitude of the incident wave vector. The simulation curve is offset vertically by 0.2 and corresponds to the vertical scale labeled on the right axis in Fig. 2.

In the simulation, we adjusted the blaze angle and groove geometry of the crown glass grating to match the relative intensities of the diffraction order peaks. (The details of the grating do not affect the functionality of the FL interferometer.) We used a value of $10 \mu \mathrm{m}$ for the laser focus waist in the simulation. The amplitude of the interference fringes in the Fig. 2 data is somewhat smaller than the amplitude of the simulation fringes. We attribute this to averaging effects that occurred during the integration of the fringes in the CCD image (these fringes were not perfectly vertical). The purpose of showing the simulation is to support our physical picture. The data show that with a minimum of alignment, good quality fringes can be obtained. It was not our intention to show more than qualitative agreement between the data and theory.

\section{EXPERIMENTAL RESULTS}

To measure the interference signals of the FL and Michelson interferometers, we fixed the position of photodetector PD1 (see $x_{\text {fix }}$ in Fig. 2). For the graph shown in Fig. 3(a), we modulated the audio speaker with a $3 \mathrm{~Hz}$ triangular wave (sawtooth) output of a synthesized function generator (SRS DS345). We measured and compared the interference fringes of both interferometers by observing averaged $(8 \times)$ oscilloscope traces of the signals from PD1 and PD2. A photographic film diffraction grating with 100 lines $/ \mathrm{mm}(d$ $=10 \mu \mathrm{m}$ ) was used to create the FL interferometer fringes shown in Fig. 3. We used this grating to demonstrate that one can successfully use this technique with low-quality gratings.

In Fig. 3(a), successive interference fringes of the FL and Michelson interferometers are separated by $10 \mu \mathrm{m}$ and $\lambda_{\text {opt }} / 2=266 \mathrm{~nm}$, respectively. The Michelson fringes were used to calibrate the $x$ scale of these plots. This confirmed that the separation of the FL interferometer fringes was indeed $10 \mu \mathrm{m}$. The insets show an expanded view of the Michelson fringes for the indicated region. We fit the fringes in Figs. 3(a) and 3(b) with sinusoidal functions, but the sinusoidal fit curves are not shown so that the figures are easier to view. (It should be noted that the audio speaker response to the drive voltages used in our experiments is dominantly linear.)

In the Fig. 3(b) plots we introduced mechanical vibration into the modulation of the audio speaker by combining the 
(a) Focused-Laser Interferometer

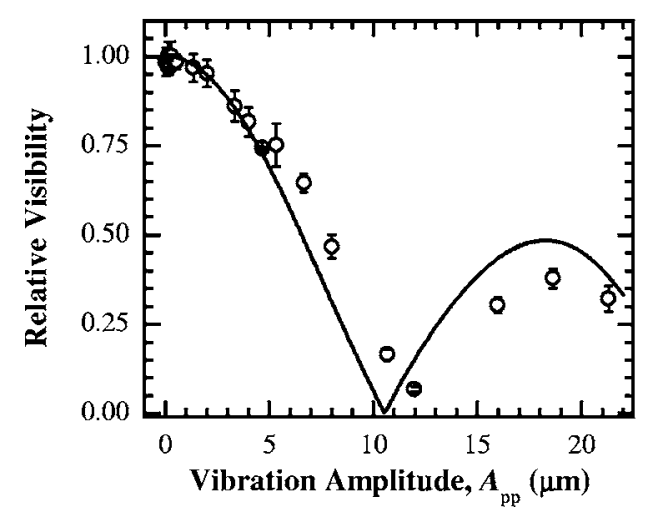

(b) Michelson Interferometer

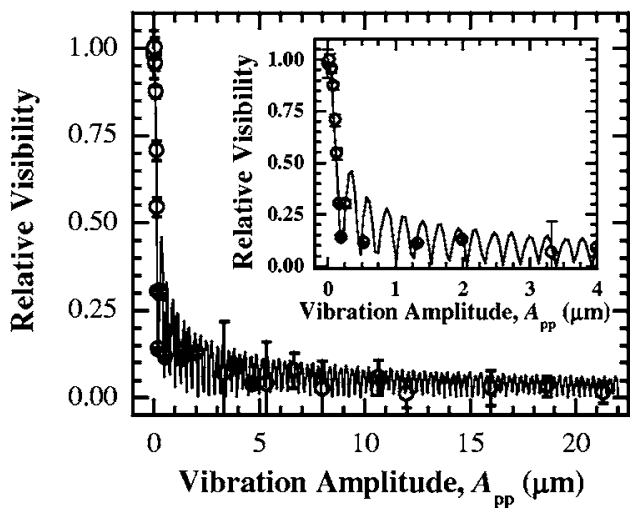

FIG. 4. Plots of the relative visibility of our focused-laser interferometer (a) and our Michelson interferometer (b) vs the peak-to-peak vibration amplitude $A_{\mathrm{pp}}$ of a crown glass diffraction grating and the mirror HR1. The vibration amplitude was caused by a $60 \mathrm{~Hz}$ sinusoidal signal being added to the $2.456 \mathrm{~Hz}$ sawtooth modulation of the audio speaker. The solid lines in (a) and (b) are numerical simulations of the data. The inset in (b) shows the relative visibility of the Michelson interferometer, for vibration amplitudes ranging from $A_{\mathrm{pp}}=0$ to $A_{\mathrm{pp}}=4 \mu \mathrm{m}$.
$3 \mathrm{~Hz}$ sawtooth modulation with the white-noise output of a second DS345 function generator. We combined and amplified the two signals with a low-noise preamplifier (SRS SR560). We fed the output of the preamplifier into a homebuilt voltage to current $(V-I)$ amplifier. The $V-I$ amplifier provided the current needed to move the speaker membrane a maximum distance of approximately $20 \mu \mathrm{m}$ during one slope of the $3 \mathrm{~Hz}$ sawtooth modulation. The plots in Fig. 3(b) show the fringes of both interferometers when $0.75 \mathrm{~V}$ (rms amplitude) of white noise was added to the speaker modulation. Note the change of the fringes from Fig. 3(a) to 3(b). The interference amplitude and the sinusoidal shape of the Michelson fringes are nearly destroyed in Fig. 3(b). However, the amplitude and shape of the FL interferometer fringes are just beginning to show the effects of the white noise in Fig. 3(b).

To make a detailed comparison of the sensitivity of FL and Michelson interferometers to mechanical vibration, we measured the visibility of both interferometers when a $60 \mathrm{~Hz}$ sinusoidal signal was added to the sawtooth modulation of the speaker. The sawtooth modulation frequency was $2.456 \mathrm{~Hz}$ for this experiment. We use Michelson's definition of visibility $V \equiv\left(I_{\max }-I_{\min }\right) /\left(I_{\max }+I_{\min }\right)$, where $I_{\max }$ and $I_{\min }$ are maximum and minimum intensities (see p. 556 of Ref. 11). We chose a $60 \mathrm{~Hz}$ frequency because it is a common frequency of mechanical vibration found in many experiments. The crown glass diffraction grating $(d=14 \mu \mathrm{m})$ was used in the FL interferometer for this experiment.

The $60 \mathrm{~Hz}$ sinusoidal signals ranged in amplitude (peakto-peak) from 0 to $16.0 \mathrm{~V}_{\mathrm{pp}}$. For each $60 \mathrm{~Hz}$ voltage that was added to the $2.456 \mathrm{~Hz}$ speaker modulation, interference fringes of both interferometers were fitted with a sinusoidal curve. The maximum and minimum values of the individual sinusoidal fits $\left(I_{\max }\right.$ and $\left.I_{\min }\right)$ were used to calculate the relative visibility of both interferometers for each $60 \mathrm{~Hz}$ voltage. At $0 \mathrm{~V}$ we normalized the visibility to 1 . To set the horizontal scale of both graphs in Fig. 4, we counted the number of Michelson fringes created by $60 \mathrm{~Hz}$ signals ranging from 0 to $8.0 \mathrm{~V}_{\mathrm{pp}}$ (with the $2.456 \mathrm{~Hz}$ sawtooth modulation turned off). This calibrated the peak-to-peak vibration amplitude $\left(A_{\mathrm{pp}}\right)$ of the grating and HR1 with the $60 \mathrm{~Hz}$ voltage amplitude $\left(V_{\mathrm{pp}}\right)$ input to the audio speaker. A linear fit of this data gave a calibration of $A_{\mathrm{pp}}=(1.3 \pm 0.2) \mu \mathrm{m} / V_{\mathrm{pp}}$. The round symbols in Figs. 4(a) and 4(b) show the relative visibility of the FL and Michelson interferometers versus the vibration amplitude $A_{\mathrm{pp}}$. A relative visibility of 1.0 corresponds to an absolute visibility of 0.26 for the FL interferometer and 0.55 for the Michelson.

The solid lines in Figs. 4(a) and 4(b) are numerical simulations of the relative visibility $V\left(A_{\mathrm{pp}}\right)$ of each interferometer as a function of vibration amplitude. The functions shown in Eqs. (1) are the maximum $I_{\max }\left(A_{\mathrm{pp}}\right)$ and minimum $I_{\min }\left(A_{\mathrm{pp}}\right)$ intensities used to calculate $V\left(A_{\mathrm{pp}}\right)$. In Eqs. (1) $\nu_{1}$ $=2.456 \mathrm{~Hz}$ and $\omega_{1}=2 \pi \nu_{1} ; \nu_{2}=60 \mathrm{~Hz}$ and $\omega_{2}=2 \pi \nu_{2} ; B$ is a constant background; and $k=2 \pi / \lambda$, where $\lambda$ is either the $d$ $=14 \mu \mathrm{m}$ periodicity of the crown glass grating used in the FL interferometer in Fig. 4(a) or half of the laser wavelength $\left(\lambda_{\text {opt }} / 2=266 \mathrm{~nm}\right)$ that determined the periodicity of the Michelson interferometer in Fig. 4(b). To calculate the maximum and minimum intensities in Eqs. (1), we integrated over a half-period of the $60 \mathrm{~Hz}$ sinusoidal signal, i.e., from $t=0$ to $t=1 /\left(2 \nu_{2}\right)$. The maximum intensities $I_{\max }\left(A_{\mathrm{pp}}\right)$ occur when the temporal phase of the $2.456 \mathrm{~Hz}$ sawtooth modulation is $\omega_{1} t=0$. The minimum intensities $I_{\min }\left(A_{\mathrm{pp}}\right)$ occur when the temporal phase is $\omega_{1} t=\pi / \omega_{1}$.

$$
\begin{aligned}
& I_{\max }\left(A_{\mathrm{pp}}\right) \propto \int_{0}^{1 /\left(2 \nu_{2}\right)} B+\cos ^{2}\left\{k\left[\left(A_{\mathrm{pp}} / 2\right) \cos \left(\omega_{2} t\right)\right]+0\right\} d t, \\
& I_{\min }\left(A_{\mathrm{pp}}\right) \propto \int_{0}^{1 /\left(2 \nu_{2}\right)} B+\cos ^{2}\left\{k\left[\left(A_{\mathrm{pp}} / 2\right) \cos \left(\omega_{2} t\right)\right]+\pi / \omega_{1}\right\} d t .
\end{aligned}
$$

In Fig. 4(a) there is a roughly $12 \%$ error between the data and the simulation. The error is related to the uncertainty of the calibration of the vibration amplitude with the amplitude of the $60 \mathrm{~Hz}$ signal that was input to the audio speaker. This uncertainty was likely caused by a nonlinear response of the speaker membrane due to the added load of the grating and HR1. The inset of Fig. 4(b) is an expanded view of the Michelson interferometer relative visibility for vibration amplitudes ranging from $A_{\mathrm{pp}}=0$ to $A_{\mathrm{pp}}=4 \mu \mathrm{m}$. The inset confirms that the relative visibility of the Michelson fringes falls off at roughly the expected rate.

It is clear in Fig. 4 that our FL interferometer is much less sensitive to mechanical vibration than the Michelson. The relative visibility of the FL interferometer simulation curve equals 0.5 when $A_{\mathrm{pp}}=7 \pm 0.9 \mu \mathrm{m}$, while the relative visibility of the Michelson simulation curve equals 0.5 when 
$A_{\mathrm{pp}} \approx 120 \mathrm{~nm}$. The ratio of these amplitudes, $(7 \pm 0.9) \mu \mathrm{m} / 120 \mathrm{~nm}=58 \pm 7$, is close to the ratio of the interferometer periodicities $14 \mu \mathrm{m} / 266 \mathrm{~nm} \approx 53$. This highlights the fact that a FL interferometer can be used as a position sensor when it is not possible to use a Michelson.

\section{DISCUSSION}

A focused-laser interferometric position sensor is a useful tool to measure or monitor the position shifts of an object. This sensor is much less sensitive to mechanical vibration than a Michelson interferometer, and it has the same spatial resolution as a three-grating MZ interferometer. The ability of a FL position sensor to accurately measure or monitor an object's position is limited by the amplitude of the object's vibration. However, one can use a diffraction grating with a larger periodicity in the case of larger vibration amplitudes. There will be a loss of spatial resolution associated with a larger grating periodicity but, nonetheless, the resolution may be more than adequate. The working distance of a FL position sensor can be changed by changing the focal length of the last lens. One must be careful to use a lens geometry that causes the diffraction orders to overlap at the desired working distance. The condition is that the laser beam divergence must be greater than the diffraction angle. Finally we note that if the working distance between the grating and the last lens is varied, the spatial separation of the interference fringes changes. However, as the grating moves, the oscillation period of the fringes is independent of the working distance. For example, with the lens configuration used in our experiments we could use distances of
$15 \pm 1.5 \mathrm{~cm}$ between the grating and the $f=10 \mathrm{~cm}$ cylindrical lens. Also the relative tilt between the grating and the cylindrical lens is not very sensitive. Thus this interferometer is easy to align.

\section{ACKNOWLEDGMENTS}

The authors wish to thank Nathan Chandler-Smith and Shanmugatharasu Periyannan for technical assistance. The authors gratefully acknowledge the financial support of the National Science Foundation and DOD-EPSCoR.

${ }^{1}$ H. Fizeau, Ann. Chim. Phys. 66, 429 (1862).

${ }^{2}$ H. Fizeau, Acad. Sci., Paris, C. R., Paris, C. R. 66, 934 (1868).

${ }^{3}$ M. Born and E. Wolf, Principles of Optics, 7 th (expanded) ed. (Cambridge University Press, Cambridge, England, 2003).

${ }^{4}$ A. A. Michelson, Am. J. Sci. 22, 120 (1881).

${ }^{5}$ A. A. Michelson, Philos. Mag. 13, 236 (1882).

${ }^{6}$ L. Zehnder, Z. Instrumentenkd. 11, 275 (1891).

${ }^{7}$ L. Mach, Z. Instrumentenkd. 12, 89 (1892).

${ }^{8}$ C. Fabry and A. Perot, Ann. Chim. Phys. 16, 115 (1899).

${ }^{9}$ M. G. Sagnac, Acad. Sci., Paris, C. R., Paris, C. R. 152, 310 (1911).

${ }^{10}$ M. G. Sagnac, Acad. Sci., Paris, C. R., Paris, C. R. 157, 708, 1410 (1913).

${ }^{11}$ E. Hecht, Optics, 3rd ed. (Addison Wesley Longman, New York, 1998), pp. 399-405.

${ }^{12}$ G. Cavagnero, G. Mana, and E. Massa, Rev. Sci. Instrum. 76, 053106-1 (2005).

${ }^{13}$ H. Stiebig, H. Büchner, E. Bunte, V. Mandryka, D. Knipp, and G. Jäger, Appl. Phys. Lett. 83, 12 (2003).

${ }^{14}$ S. Dong, F. Bai, J. Li, and D. Viehland, Rev. Sci. Instrum. 74, 4863 (2003).

${ }^{15}$ A. Miffre, R. Delhuille, B. Viaris de Lesegno, M. Büchner, C. Rizzo, and J. Vigué, Eur. J. Phys. 23, 623 (2002).

${ }^{16} \mathrm{~J}$. Moromisato, S. Reucroft, R. Terry, E. von Goeler, D. Eartly, and K. Maeshima, Nucl. Instrum. Methods Phys. Res. A 538, 234 (2005).

${ }^{17}$ A. Yariv, Optical Electronics, 4th ed. (Saunders College Publishing, Orlando, 1991), pp. 46-48. 